\title{
Comparative analysis of methods for inferring successful foraging areas from Argos and GPS tracking data
}

\author{
Anne-Cécile Dragon ${ }^{1,2,3, *}$, Avner Bar-Hen ${ }^{2}$, Pascal Monestiez ${ }^{4}$, Christophe Guinet ${ }^{1}$ \\ ${ }^{1}$ CEBC-CNRS, 79360 Villiers en Bois, France \\ ${ }^{2}$ MAP5-CNRS, 45 rue des Saints-Pères, 75270 Paris Cedex 06, France \\ ${ }^{3}$ LOCEAN-UPMC, 4 place Jussieu, boite 100, 75252 Paris Cedex 05, France \\ ${ }^{4}$ INRA, UR546 Biostatistique et Processus Spatiaux (BioSP), 84914 Avignon, France
}

\begin{abstract}
Identifying animals' successful foraging areas is a major challenge, but such comprehensive knowledge is needed for the management and conservation of wild populations. In recent decades, numerous specific analytic methods have been developed to handle tracking data and to identify preferred foraging areas. In this study, we assessed the efficiency of different track-based methods on Argos and GPS predators' tracks. We investigated (1) the consistency in the detection of foraging areas between track-based methods applied to 2 tracking data resolutions and (2) the similarity of foraging behaviour identification between track-based methods and an independent index of foraging success. We focused on methods that are commonly used in the literature: empirical descriptors of foraging effort, Hidden Markov Models (HMMs) and first passage time analysis. We applied these methods to satellite tracking data collected on 6 long-ranging elephant seals equipped with both Argos and GPS tags. Seals were also equipped with time depth recorder loggers from which we estimated an independent index, based on the drift rate and the changes in the seals' body condition, as a proxy for foraging success along the tracks. Favourable foraging zones identified by track-based methods were compared to locations where the body condition of the seals significantly increased. With or without an environmental covariate, HMMs were the most reliable for identifying successful foraging areas on both high (GPS) and low (Argos) resolution data. Areas identified by HMMs as intensively used were congruent with the locations where seals significantly increased their body condition given a $4 \mathrm{~d}$ metabolisation lag.
\end{abstract}

KEY WORDS: Area-restricted-search - Drift dive - First bottom time - Mirounga leonine . Movement analysis $\cdot$ State-space modelling

Resale or republication not permitted without written consent of the publisher

\section{INTRODUCTION}

In natural habitats, individual fitness directly depends on animals' ability to acquire resources in an efficient way (Stevick et al. 2002). While foraging, predators often display movement patterns at multiple spatial and temporal scales that are assumed to match the spatial structure of their prey environment (Fauchald 1999). Therefore, it is expected that movement analysis can reveal profitable patches used by an animal (Turchin 1991). In a prey-aggregated environment such as the open ocean, it is pre- dicted that a predator having already captured a first prey would intensify its foraging in the patch (Charnov 1976, Parker \& Stuart 1976). This results in a behaviour called the area-restricted search (ARS, Kareiva \& Odell 1987) and is defined by a decrease in speed and an increase in sinuosity of movement (Benhamou \& Bovet 1989). Between 2 patches, on the other hand, the forager travels more linearly and at a faster pace. Therefore, track-based measurements of free-ranging predators are thought to provide information on their foraging behaviour. 
In recent decades, technical advances in wildlife telemetry have enabled the study of animals' movement patterns in both the terrestrial and marine environments (Buechner et al. 1971, White \& Garrott 1990, Block et al. 2001). Increasingly, satellite telemetry (using the Argos system) and GPS (Global Positioning System) technologies yield high quality tracking time series. For both Argos and GPS devices, however, batteries have a limited lifespan and are designed to last for a specific number of acquisitions and/or transmissions. Argos devices allow the collection and emission by satellite of month-long, but noisy, recordings. This data resolution, low sampling frequency and low spatial resolution, will be referred to in the manuscript as the Argos data resolution. On the other hand, GPS devices combine a very high sampling frequency with a high spatial resolution (95\% of records with an uncertainty lower than $50 \mathrm{~m}$ ). However, the life expectancy of GPS devices is short and generally does not allow data transmissions by satellite, making it necessary to retrieve the GPS device to obtain high resolution tracking data. This compromise, typically between accurate, frequent and power-consuming GPS locations and less precise, less frequent but longer recordings of Argos locations, has direct implications for tracking data analysis.

Filtering of measurement errors, for Argos locations, and detection of changes in movement and foraging behaviour are 2 main issues in tracking data analyses. Recently, a growing number of analytical methods have been developed to deal with one or both of these issues (Fauchald \& Tveraa 2003, Buckland et al. 2004, Jonsen et al. 2005, Patterson et al. 2010, among others). To identify intensively used areas along predators' tracks 2 types of approaches can be distinguished: descriptive methods and process-based models. In the ARS context, empirical descriptors, such as step length or turning angles, play a key role in detecting intensive foraging areas (Benhamou \& Bovet 1989, Bartumeus et al. 2008). Considering a diving predator that forages and feeds in the water column, the number of dives per kilometre can also be an indicator of foraging intensity: the higher the number of dives in a given distance, the more intense the foraging of the predator (Dragon et al. 2010). The proportion of the dive spent at the bottom has also been shown to be an indicator of foraging activity in several diving predators, with animals spending more time at the bottom in favourable foraging conditions (elephant seal Mirounga leonina: Boyd \& Arnbom 1991; sperm whale Physeter macrocephalus: Miller et al. 2004; grey seal Halichoerus grypus: Austin et al. 2006; Australian sea lion Neophoca cinerea: Fowler et al. 2006).
Furthermore, maximum diving depth can indicate the animal's energetic budget: the deeper the dives in the water column, the more expensive the access to resources and the less time the animal can spend at the bottom (Le Boeuf 1994). Positive residuals from a multiple regression (bottom time as a function of maximum diving depth and dive duration) indicate longer bottom time than expected for a given diving depth and duration (Bailleul et al. 2007b). Therefore, higher bottom time residuals (resBT) correspond to increased foraging time at the bottom of the dive (Bailleul et al. 2007b). Other specific descriptive track-based analyses were recently published, such as fractal analysis, where a segment of a given length is moved along the animal track and the fractal dimension is calculated for each segment (Dicke \& Burrough 1988, Schmitt \& Seuront 2001, Tremblay et al. 2007). The ARS are identified by high track convolutions corresponding to an increased fractal dimension. Intensively used areas are identified by high values in the sinuosity distribution displayed by one of the random walks. Finally, first passage time (FPT) provides a measure of the time an animal takes to cross a virtual circle of a radius $r$ that is moved along its track (Fauchald \& Tveraa 2003). The underlying hypothesis is that high FPT in certain areas corresponds to an ARS display. Bailleul et al. (2008) adapted FPT to the resBT of diving predators. Their method, the first bottom time (FBT), identifies ARS along diving predators' tracks.

These descriptive methods may accurately detect the major ARS zones but lack a predictive capacity to statistically categorize distinct foraging behaviours. Recently, behavioural change point analysis has allowed the detection of behavioural changes in movement tracks with irregular measurement intervals (Gurarie et al. 2009). Mechanistic models, such as process-based models, allow a functional relationship between the system's probability of being in a state at time $t$ and the previous system states. In an $n$ th-order Markov chain, the process model probabilistically predicts the future system state from its $n$ previous states (Cappé et al. 2005). Animal movements can be modelled as correlated (and/or biased) random walks (Turchin 1991) that generally correspond to first-order Markov chains (Patterson et al. 2008). In particular, state-space models combine an observation model to a process model (review in Patterson et al. 2008 and Schick et al. 2008). The observation model describes mathematically how observations of the states (for instance potential locations including measurement errors) are related to the real states (e.g. true locations that are unobservable because of imperfect observation technology). Unlike 
state-space models where the unobservable state lies on a continuum, Hidden Markov Models (HMMs) are process-based models with a finite set of discrete categorical hidden states (Cappé et al. 2005). For instance, the state at time $t$ can depend on a hidden variable such as the foraging mode of a free-ranging predator (Morales et al. 2004). When analysing noisy tracking data, an HMM that infers the animal's behavioural modes can be nested within a statespace model for the estimation of the animal's locations (Jonsen et al. 2005, Jonsen et al. 2007). For filtered Argos locations or very low-error GPS data, HMM assign behavioural modes directly from inferences on movement data (Franke et al. 2004, Morales et al. 2004, Vermard et al. 2010, Walker \& Bez 2010). The use of environmental covariates in the HMM inference can help in understanding how predators adapt their foraging behaviour to local environmental conditions (Morales et al. 2004). For instance, numerous marine predators have shown preferences for oceanographic features that could be quantified in the HMM context (Bost et al. 2009). Furthermore, the addition of behavioural covariates, such as resBT for a diving predator, can potentially improve the foraging mode estimation. Ultimately, the choice of method depends on the predators' ecology (its distance, regularity and scale of movement), the resolution of the collected data and on the methods' appropriateness. Foraging ecologists are confronted by this choice while having little information on the methods' abilities, for a given data resolution, to properly detect successful foraging activity.

Wide-ranging predators are often impossible to observe across their range, which makes the estimation of their foraging success more difficult. In the case of diving predators, though, their body condition depends on the animals' buoyancy, which is determined by the relative proportions of lipids and lean body tissue (Webb et al. 1998). Therefore, buoyancy modifications along a diving predator's track indicate variations in body condition that are directly related to its foraging success. In particular, a predator species that drifts passively in the water column while diving provides an ideal opportunity to infer in situ body condition (Biuw et al. 2003). Travelling thousands of kilometres per year in the circumpolar waters of the Southern Ocean (McConnell et al. 1992), southern elephant seals Mirounga leonina are elusive marine predators. They continuously dive during their extended stay at sea and display behaviours named 'drift dives' along their tracks. Southern elephant seals regularly perform dives during which they stop swimming and drift passively in the water column (Biuw et al. 2003). Vertical movements during these dives are related to the seal's body condition (Webb et al. 1998): fat and positively buoyant seals will follow an upward drift. Inversely, lean seals with a negatively buoyant body condition will sink during a drift dive. An increase in the drift rate over time indicates recent lipid anabolism and the occurrence of a previous successful foraging activity (Bailleul et al. 2007b, Biuw et al. 2007). This drift rate index provides a rare independent measure to evaluate the foraging zones identified using the trackbased methods available to foraging ecologists.

Our aims in this study were 2-fold: we investigated (1) the similarity of several track-based methods to an independent index of foraging success and (2) assessed the influence of data resolution to determine the most adequate method for the identification of successful foraging areas. Among the track-based methods previously mentioned, fractal dimension is still controversial (Turchin 1996, Benhamou 2004), and behavioural change point analysis detects the behavioural changes but cannot ascertain whether there is a finite number of behavioural modes and where they occur. We thus decided to focus our study on the most commonly used methods for diving predators. Therefore, we tested, on GPS data and previously filtered Argos data, 4 traditional track descriptors, FPT and FBT methods and finally various HMM, with and without environmental covariates. We investigated, for these different track-based methods, the similarity of ARS identification between the analyses of elephant seals' GPS and filtered Argos tracks. We then evaluated, for each data resolution, the validity of ARS identification by the trackbased methods against an independent dive-based measure of foraging success, accounting for an estimated time lag of metabolisation. A better assessment of the proper detection of successful foraging and of the effect of data resolution on track-based methods will provide guidelines for future foraging studies.

\section{MATERIALS AND METHODS}

\section{Tracking data and diving behaviour}

\section{Deployment of devices and data processing}

Post-breeding southern elephant seal females were captured, in October $2008(\mathrm{n}=2)$ and $2009(\mathrm{n}=5)$ on Kerguelen Island $\left(49^{\circ} 20^{\prime} \mathrm{S}, 70^{\circ} 20^{\prime} \mathrm{E}\right)$. Animals were anaesthetised using a 1:1 combination of Tiletamine and Zolazepam (Zoletil 100) injected intravenously 

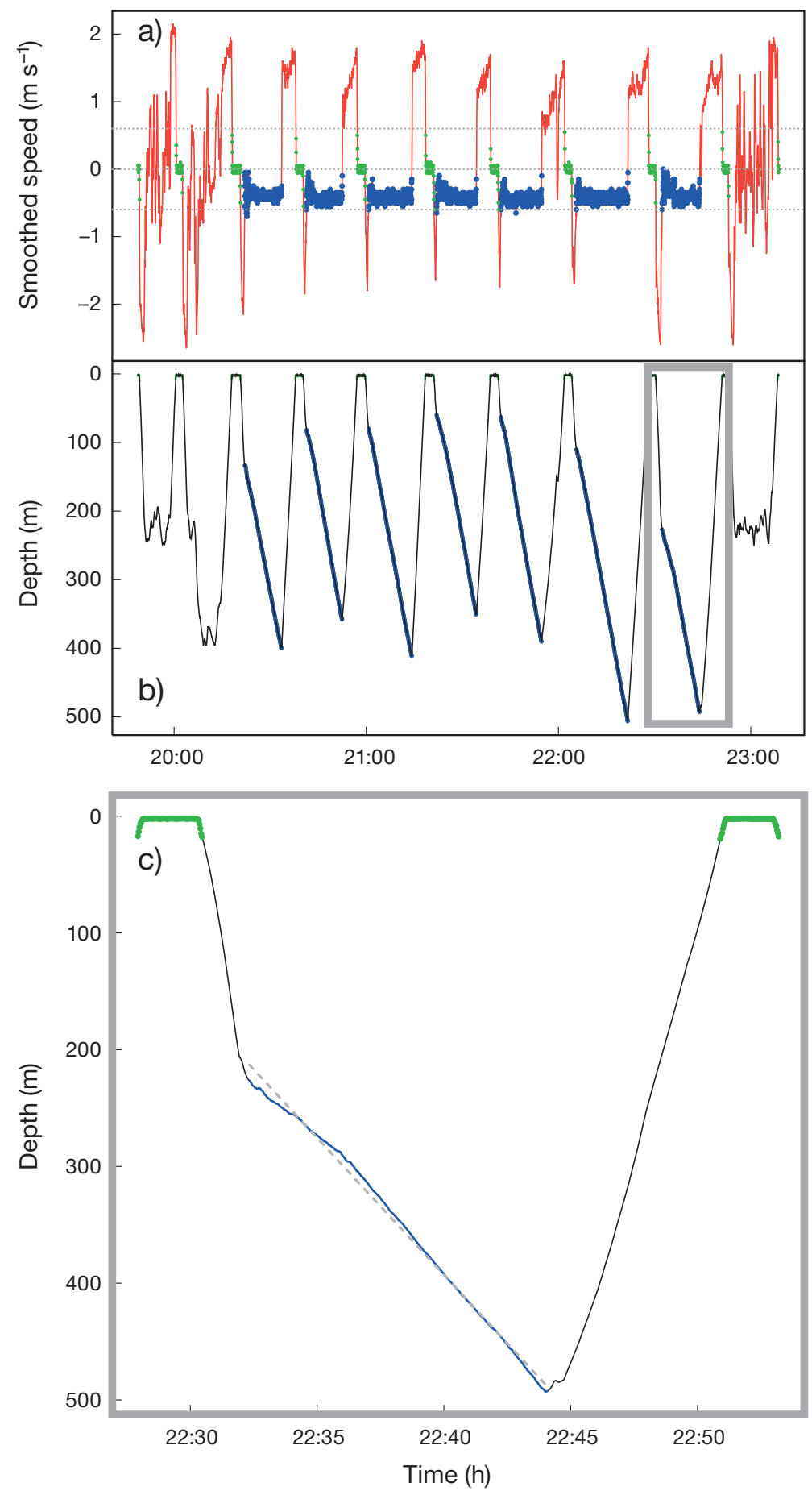

Fig. 1. Drift-dive detection process: time series of (a) smoothed vertical speed $\left(\mathrm{m} \mathrm{s}^{-1}\right)$ of southern elephant seals and (b) depth $(\mathrm{m})$. The example illustrates the detection of a set of negative drift dives on November 2, 2008, with passive swimming sections in blue and surface phases in green. (c) Example of a negative drift dive (grey frame in b). The surface time is in green and the bottom phase includes a long negative drift phase (blue with a vertical speed between -0.6 and $0 \mathrm{~m} \mathrm{~s}^{-1}$ ). A linear regression (dashed grey line) is calculated during the drift phase. The slope coefficient of the regression corresponds to the drift rate (here $-0.77 \mathrm{~m} \mathrm{~s}^{-1}$ ) associated with each drift phase
(McMahon et al. 2000). After cleaning the hair with acetone, satellite relayed data loggers (MK10 Fast-Loc, $105 \times 60 \times 20 \mathrm{~mm}$, $240 \mathrm{~g}$, with cross-sectional area $12 \mathrm{~cm}^{2}$, Wildlife Computers) were glued on the seals' heads, using quick-setting epoxy (Araldite AW 2101). The loggers collected and transmitted both Argos and GPS location data (see Tables S1 \& S2 in Supplement 1 at www.int-res.com/articles/suppl/ m452p253_supp/ that present characteristics of both resolutions). The time depth recorders (TDR) device collected pressure every 2 s. The time elapsed between 2 GPS locations was set to be a minimum of $20 \mathrm{~min}$. Six of the 7 equipped females were recovered at the beginning of the moulting season (January 2009 and 2010), and the high resolution diving data sets were downloaded.

The Argos locations of the poorest quality (Classes B and Z) were discarded. The speed between successive remaining locations was computed, and locations leading to values $>3 \mathrm{~m} \mathrm{~s}^{-1}$ were discarded (Girard et al. 2006, Dragon et al. 2010). To filter out the Argos location error, tracks were then smoothed and resampled at $6 \mathrm{~h}$ intervals using an Epanechnikov filter with a time window of $2 \mathrm{~d}$ (Gaspar et al. 2006). TDR data were processed with a standard zero-offset correction to take into account shifts in the pressure transducer calibration of the instrument over the period of data collection. Only dives deeper than $15 \mathrm{~m}$ and lasting more than 3 min were kept for analysis. For Argos and GPS tracks, each tracking location was then associated with the dive profile, a subset of the TDR data.

\section{Drift dive detection and assessment of the seals' body conditions}

Drift dive identification was processed from the complete time-depth (TDR) data as follows. First, instantaneous vertical speed was calculated from the timedepth data. It was then smoothed out by using a moving average (10 s window) in order to compensate for abrupt changes in depth reading due to the captor accu- 
racy $( \pm 1 \mathrm{~m})$. Within dives, drift phases were isolated using a custom-made function under $\mathrm{R}$ software ( $\mathrm{R}$ Development Core Team 2009). Drift phases were detected as periods of time of more than 3 min during which the vertical speed was bounded between -0.6 and $0.6 \mathrm{~m} \mathrm{~s}^{-1}$ and with a low variance $\left(\mathrm{s}^{2}<0.005\right)$ (Fig. 1, for more details see the annotated $R$ codes in Supplement 2 at www.int-res.com/articles/suppl/ m452p253_supp/).

For each drift dive, a drift rate was determined as the slope coefficient of a linear regression between depths and time (see Bailleul et al. 2007a and our Fig. 1). Daily drift rate was then calculated along the 3 mo tracks (see Fig. S1 in Supplement 1). Considering the global fattening-up trend of the seals along their tracks, we differentiated the daily drift rate to detect extreme changes in drift rate (90\% quantile). Along each individual track, a drift rate categorical variable indicated the presence of successful foraging, e.g. where the seals improved greatly in body condition and buoyancy (Crocker et al. 1997, Biuw et al. 2003, Bailleul et al. 2007b).

\section{Track-based methods for the detection of foraging areas}

\section{Empirical descriptors of tracking data}

We worked on both data resolutions to detect intensive foraging areas from simple track metrics. Four empirical descriptors of seal tracks were calculated: step length (the net distance moved between 2 location measurements), number of dives (between 2 locations), bottom time residuals and maximum diving depth. In the ARS context, we expected to detect intensive foraging events when the seals displayed small step length (Biuw et al. 2007), high number of dives per kilometre, small dive depth and high bottom time residuals. Therefore, 4 categorical variables indicated the presence of intensive foraging areas from the extreme values in step length $(<10 \%$ quantile), number of dives $(>90 \%$ quantile), bottom time residuals (>90\% quantile) and maximum diving depth $(<10 \%$ quantile).

First passage time and first bottom time

FPT refers to the period of time that an animal, supposed to be following a random walk, takes to leave a circle centred on its location at time $t$ (Fauchald \& Tveraa 2003). High FPT indicates ARS behaviour dis- played at the spatial scale $S$, the chosen radius of the circle that corresponds to the maximum peak in FPT relative variance (see Fauchald \& Tveraa 2003). For each individual, we used FPT and its derived method (FBT) to analyse the Argos and GPS tracking data. As identified in preliminary studies (not presented here; see Bailleul et al. 2008 for methodological details), $S$ used for FPT and FBT analyses on Argos tracks was $49 \pm 32 \mathrm{~km}$ (mean $\pm \mathrm{SD}$ ) and $57 \pm 23 \mathrm{~km}$, respectively. $S$ on GPS tracks was $36 \pm 16 \mathrm{~km}$ (FPT) and $60 \pm 47 \mathrm{~km}$ (FBT). Along the paths, FPT and FBT categorical variables indicating the presence of ARS were defined by long FPT/FBT times (90\% quantile) determined at $S$.

\section{Hidden Markov Models}

In this study, we used 4 HMMs developed by Morales et al. (2004) with 2 hidden, or unobservable, behavioural modes and an increased number of covariates. HMMs estimate the probability $p[t, \mathrm{~b}$. mode] of each observation point, in our case a vector of 2 components that are step length and turning angle calculated from tracking data at time $t$, to be in the 2 different behavioural modes b.mode $\in\{1,2\}$ (Eq. 1). At each time step, an individual can change from the current behavioural mode to a different one with an estimated probability $q$ as a function of time and the previous behavioural mode. For 2 possible behavioural modes, the model estimates a $2 \times 2$ probability matrix (for details see the annotated BUGS code in Supplement 2). Model parameters are estimated, in each behavioural mode, from specific distributions (Weibull distribution for step length and wrapped Cauchy for turning angles; for details see Morales et al. 2004).

$$
\left\{\begin{array}{l}
p[t, 1]=q\left[t, \mathrm{~b} \cdot \text { mode }_{t-1}\right] \\
p[t, 2]=1-q\left[t, \text { b.mode }_{t-1}\right]
\end{array}\right.
$$

where $q$ is the probability function that describes the probability of being in an intensive foraging mode [b.mode $=1$ ] at time $t$ depending on the previous behavioural mode (b.mode $e_{t-1}$ ).

The first HMM (HMM.0) includes no covariate to estimate the probability of being in a behavioural mode $i \in\{1,2\}$ at time $t$, given that the animal was in mode $j \in\{1,2\}$ at time $t-1$. The probability matrix is fixed in time. However, the probabilities of switching between behavioural modes can also depend on environmental and/or diving covariates.

The second model (HMM.SLA) is defined by a switching probability depending on the previous 
behavioural mode and a local environmental covariate that is likely to increase switching probability from extensive to intensive foraging mode. A logistic link was used to transform the regression with a continuous environmental covariate to a $[0,1]$ probability response:

$$
\begin{aligned}
& q\left[t, \mathrm{~b} \cdot \operatorname{mode} e_{t-1}\right]=
\end{aligned}
$$

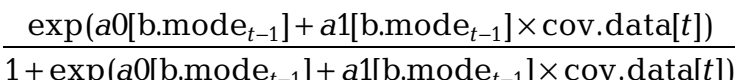

with regression coefficients $a 0$ and $a 1$ depending on the previous behavioural mode (b.mode $t_{t-1}$ ). The covariate (cov.data) is measured locally for each time step $t$.

Considering the covariate choice, previous studies have shown that mesoscale eddies, highly correlated to sea level anomalies data (SLA), were profitable foraging areas for southern elephant seals (Bailleul et al. 2010, Dragon et al. 2010). Weekly satellite maps of a third degree ground resolution of SLA were obtained from the Aviso data base (www.aviso. oceanobs.com), from which we extracted SLA values associated with each Argos and GPS track point of the individuals.

Similarly, the third HMM (HMM.resBT) depended on the bottom time residuals (resBT) as a behavioural covariate. resBT were calculated for each dive associated with an Argos or GPS track point. High resBT are assumed to increase the probability of switching from an extensive to an intensive foraging mode (Bailleul et al. 2008).

Finally, the fourth HMM model, HMM.SLA.resBT, which includes the 2 previous covariates, is intended to evaluate the relative importance of diving behaviour and environmental interactions in the foraging process:

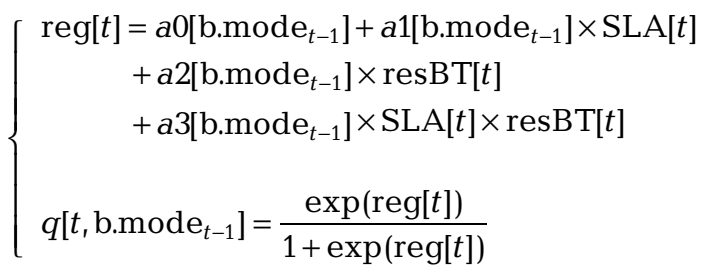

with regression (reg) coefficients $a 0, a 1, a 2$ and $a 3$ depending on the previous behavioural mode b.mode ${ }_{t-1}$. The covariates, SLA and resBT, are measured locally and calculated for each time step $t$, respectively.

Considering the number of observations, Bayesian methods become particularly useful in determining the best behavioural mode estimations. All models were fitted with freely available software winBUGS (Bayesian Analysis Using Gibbs Sampler, Spiegelhalter et al. 1999) called from $R$ (R Development Core Team 2009) with the package R2WinBUGS (Sturtz et al. 2005). For all models, we used vague priors (Gamma and Uniform distributions; for details see Supplement 2) as recommended to avoid the inherent bias of Bayesian methods as much as possible (Dennis 1996). Three MCMC (Monte-Carlo Markov Chain techniques) chains were run for each model, with 20000 iterations following a 5000 burnin (thin $=100$ ). Autocorrelation and convergence to stationary distributions were examined in sample paths of various parameters (Gelman \& Rubin 1992, Brooks \& Gelman 1998, Morales et al. 2004). The deviance information criterion (DIC, Spiegelhalter et al. 2002) was used to select between the HMM formulations. For each individual, we used the $4 \mathrm{HMMs}$ to analyse the Argos and GPS tracking data. The time series of intensive foraging probabilities $(p[t, 1])$ were transformed into categorical time series: when $p[t ; 1]>0.5$, the seal was considered to be in intensive foraging mode (1) at time $t$.

\section{Output similarity between drift rate index of foraging and analyses of Argos and GPS tracks}

\section{Similarity of foraging identification on Argos and GPS tracks}

For each individual, method and data resolution, the percentage of time allocated to intensive foraging, relative to the track durations, was calculated from the categorical time series (intensive vs. extensive foraging). Identified intensive foraging zones on Argos tracks were compared with the one identified on respective individuals' GPS tracks. Similarity between the time series was evaluated with 2 indexes: the Jaccard index (Jaccard 1901) and an index derived from the Levenshtein distance (Eq. 4) (Levenshtein 1966, Dale 1989, Papadimitriou 2009, Farina et al. 2011). This distance, also known as edit distance, is a parameter which is easy to compute which is used for measuring the amount of differences between 2 categorical time series. The greater the Levenshtein distance (LD), the greater the difference between the sequences and therefore the difference in the segmentations between the 2 sequences. Conversely, the LD similarity index is high when identifications of intensive foraging zones between the 2 time series are similar. 


$$
\left\{\begin{array}{l}
\mathrm{LD}=\frac{100 \times \sum d+i+s}{l} \\
\mathrm{LD} \cdot \mathrm{idx}=100-\mathrm{LD}
\end{array}\right.
$$

where LD is calculated from $d$ the number of deletions, $i$ the number of insertions and $s$ the number of substitutions required to transform the first categorical time series, of length $l$, into the second where LD.idx is the LD similarity index.

To evaluate the influence of data sampling frequency given an equally fine spatial resolution dataset, we progressively subsampled the GPS tracks. We worked from full GPS resolution (36 locations $\mathrm{d}^{-1}$ on average, e.g. 1 location every $40 \mathrm{~min}$ ) to Argos temporal sampling frequency (4 locations $\mathrm{d}^{-1}$ ), with intermediate resolutions $\left(18,12,9,6\right.$ locations $d^{-1}$ equally separated in time). For the Jaccard and LD similarity indexes, the similarity of allocated intensive foraging was evaluated between Argos resolution and the 6 GPS subsampled datasets.

\section{Comparison with drift rate index of foraging}

For each track-based method, successful foraging zones, identified from the drift rate index of foraging, were compared to the identified intensive foraging zones on respective individuals' Argos and GPS tracks. The similarity between track-based methods outputs and the categorical time series of drift rate index of foraging was calculated with the Jaccard and LD similarity indexes. However, to take into account the necessary time to digest and metabolise the ingested preys into seal fat, we introduced, in the similarity calculations, a time lag ranging from 0 to $12 \mathrm{~d}$ (Thums et al. 2008). We assumed that maximum similarity indexes indicated the time needed for lipid anabolism in post-breeding southern elephant seal females.

\section{RESULTS}

\section{Distribution at sea, diving and drifting behaviour}

All but one individual foraged exclusively within the interfrontal zone in pelagic waters (Fig. 2). The remaining one went to the Antarctic Plateau area. During the course of this study, animals for which tags were recovered $(\mathrm{n}=6)$ had an average foraging trip duration of $84.4 \pm 10.5 \mathrm{~d}$ (mean $\pm \mathrm{SD}$ ) and covered an average distance of $4314 \pm 979 \mathrm{~km}$. They dove on average $58.6 \pm 6.5$ times a day and displayed
$3.1 \pm 0.8$ drift dives per day. The individual that went to the Antarctic Plateau during its foraging trip performed significantly more drift dives per day than the others $(4.5 \pm 1.03$ vs. $2.8 \pm 0.4, t=-3.7, \mathrm{df}=105, \mathrm{p}=$ $0.0001)$. For the drift rate, all animals presented a positive mean rate of increase $\left(0.11 \mathrm{~m} \mathrm{~s}^{-1} \pm 0.04\right)$ over their whole foraging trip, reflecting an increase in buoyancy along their paths.

\section{Convergence and deviance information criterion of Hidden Markov Models}

The 2 first HMMs, HMM.0 and HMM.SLA, reached convergence within the burn-in phase of all individuals at both data resolutions. HMM.resBT and HMM.SLA.resBT did not converge for all individuals (Table 1). DIC was compared for all models that converged: the simplest model (no covariate) displayed the lowest DIC values (Table 1). For HMM.SLA, slope coefficients (a1) were marginally different between behavioural modes on Argos tracking data (slope coefficient when intensive foraging mode at time $t-1: 1.76 \pm 0.91$ and when extensive foraging mode at $t-1: 0.92 \pm 0.57, t=1.9$, $\mathrm{df}=8.4, \mathrm{p}$-value $=0.09$ ). However on GPS tracking data, no significant difference of coefficient a1 was obtained between behavioural modes (intensive at $t-1: 2.03 \pm 1.61$ and extensive at $t-1: 1.42 \pm 1.09, t=$ $0.76, \mathrm{df}=8.8, \mathrm{p}$-value $=0.46$ ). However, a positive intercept coefficient $(a 0)$ was obtained for all individuals and both data resolutions when in intensive foraging mode at $t-1$ (Argos: $2.41 \pm 0.98$; GPS: 2.76 \pm 0.84 ). Therefore, the probability of staying in an intensive foraging mode was increased with negative SLA. On the contrary, the intercept coefficient was negative (Argos: $-2.53 \pm 1.05$; GPS: $-2.52 \pm$ 1.18) when the animals were in an extensive foraging mode at $t-1$.

\section{Similarity of ARS identification along GPS, subsampled GPS and filtered Argos tracks for the different track-based methods}

For GPS data, track-based methods had similar ranges of time spent on intensive foraging $(27.5 \%$ \pm 13.7 ). For Argos data, track-based methods had also similar ranges of time spent on intensive foraging $(17.1 \% \pm 15.3)$, but it was marginally more variable for Argos data resolution than for GPS $(F=3.3$, numerator $\mathrm{df}=5$, denominator $\mathrm{df}=13, \mathrm{p}$-value $=0.07$ ). Globally, the estimations of time spent on intensive 

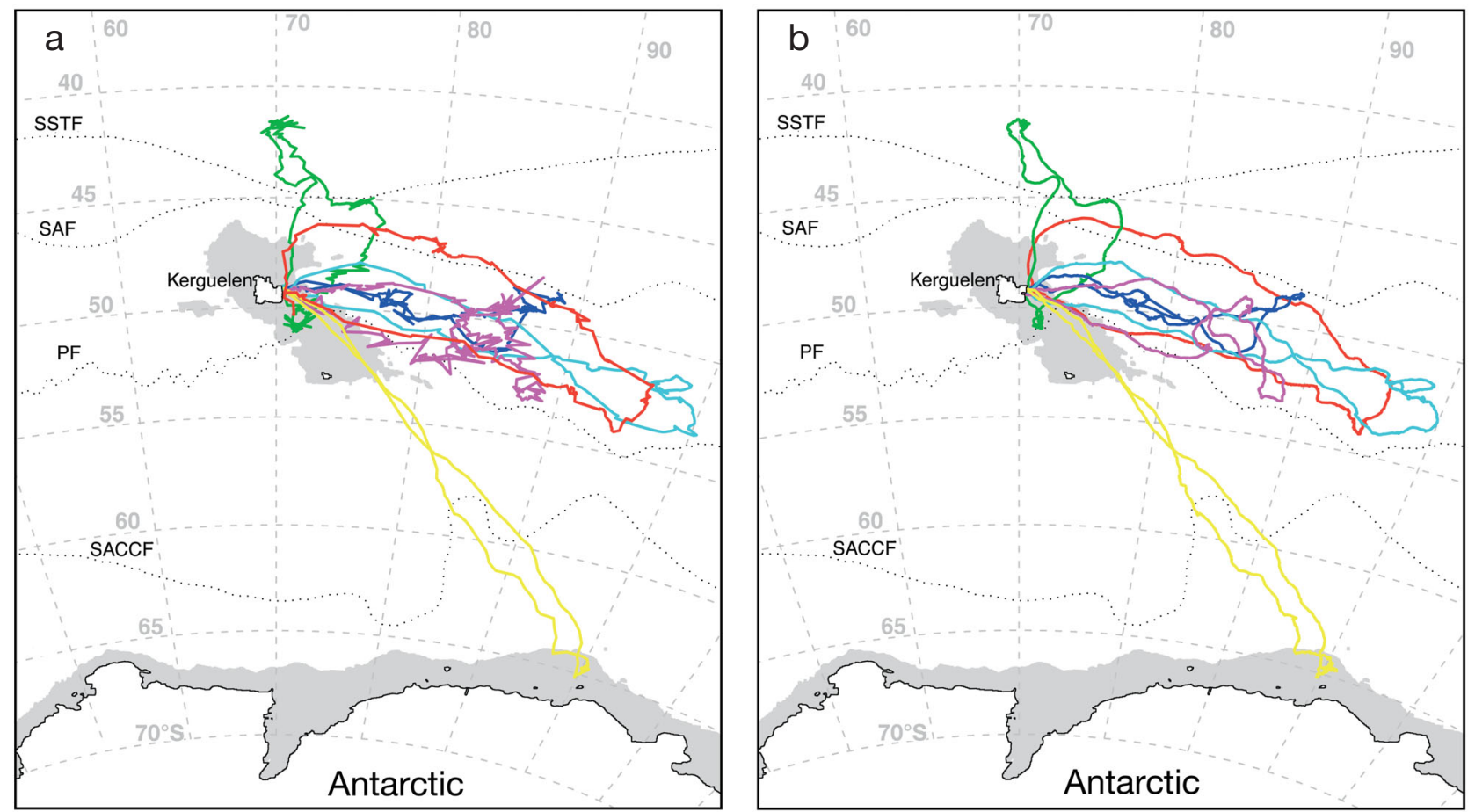

Fig. 2. (a) Filtered Argos satellite tracking and (b) GPS satellite tracking of 6 southern elephant seal females breeding on the Kerguelen Islands. Two different strategies are presented: females mainly foraged in the interfrontal zone (latitudes between 35 and $60{ }^{\circ} \mathrm{S}$ ), but 1 individual foraged in the Antarctic zone (yellow track). One female was equipped in October 2008 (red track); all other individuals in October 2009. Grey shading indicates depths $<1000 \mathrm{~m}$ (the Kerguelen Plateau and the Antarctic shelf). The Kerguelen Islands are depicted in white over the plateau. Dotted lines symbolize fronts (Orsi et al. 1995) within the Southern Ocean: Southern SubTropical Front (SSTF), Sub-Antarctic Front (SAF), Polar Front (PF) and the Southern Antarctic Circumpolar Current Front (SACCF). We refer to the interfrontal zone as the area between the SSTF and the PF

foraging were significantly different between the analyses of Argos and GPS tracks (Wilcoxon paired test: $W=88, \mathrm{p}=0.02$ ).

Fig. 3 illustrates the similarity between the outputs of track-based methods applied to Argos and on GPS tracking data (results are shown for the LD similarity index; the Jaccard index gave similar results). The 4 empirical descriptors, number of dives (Nb.Dive), resBT, maximum diving depth (Max.Depth) and step length (Step.Length) presented the highest similarities between Argos and GPS outputs. HMM.0 and
HMM.SLA also presented good similarities between the locations of intensive foraging along Argos and GPS tracks. Finally, FPT, FBT, HMM.resBT and HMM.SLA.resBT showed the lowest similarities.

Regarding variations in sampling frequency, the proportions of time allocated to intensive foraging remained nearly constant (see Fig. S2 in Supplement 1 at www.int-res.com/articles/suppl/m452p253_supp/) and standard deviations of the average percentage of time allocated to intensive foraging were very stable $(9.7 \% \pm 6.4)$.

Table 1. Deviance information criterion (DIC) for all HMM models that could reach convergence within a 5000 burn-in. Ind.: southern elephant seal individual; nc: models that did not converge

\begin{tabular}{|c|c|c|c|c|c|c|c|c|}
\hline \multirow[t]{2}{*}{ Ind. } & \multicolumn{4}{|c|}{$\longrightarrow$ Argos } & \multirow[b]{2}{*}{ HMM.0 } & \multirow[b]{2}{*}{ HMM.SLA } & \multirow{2}{*}{$\begin{array}{l}\text { _ GPS } \\
\text { HMM.resBT }\end{array}$} & \multirow[b]{2}{*}{ HMM.SLA.resBT } \\
\hline & HMM.0 & HMM.SLA & HMM.resBT & HMM.SLA.resBT & & & & \\
\hline $08-86372$ & 8892 & 14163 & 14192 & 14204 & 42585 & 85367 & $\mathrm{nc}$ & 90165 \\
\hline $09-78524$ & 3466 & 5984 & 5982 & 5985 & 43140 & 84944 & 114193 & 114815 \\
\hline $09-78525$ & 1904 & 3304 & nc & 3296 & 42813 & 84513 & $\mathrm{nc}$ & $\mathrm{nc}$ \\
\hline $09-86372$ & 2367 & 3979 & nc & nc & 54918 & 112422 & 156512 & 156967 \\
\hline $09-86373$ & 3008 & 5140 & nc & $\mathrm{nc}$ & 43498 & 86158 & 116561 & 117111 \\
\hline $09-86374$ & 3042 & 5268 & $\mathrm{nc}$ & 3296 & 41735 & 83370 & 111759 & 111810 \\
\hline
\end{tabular}




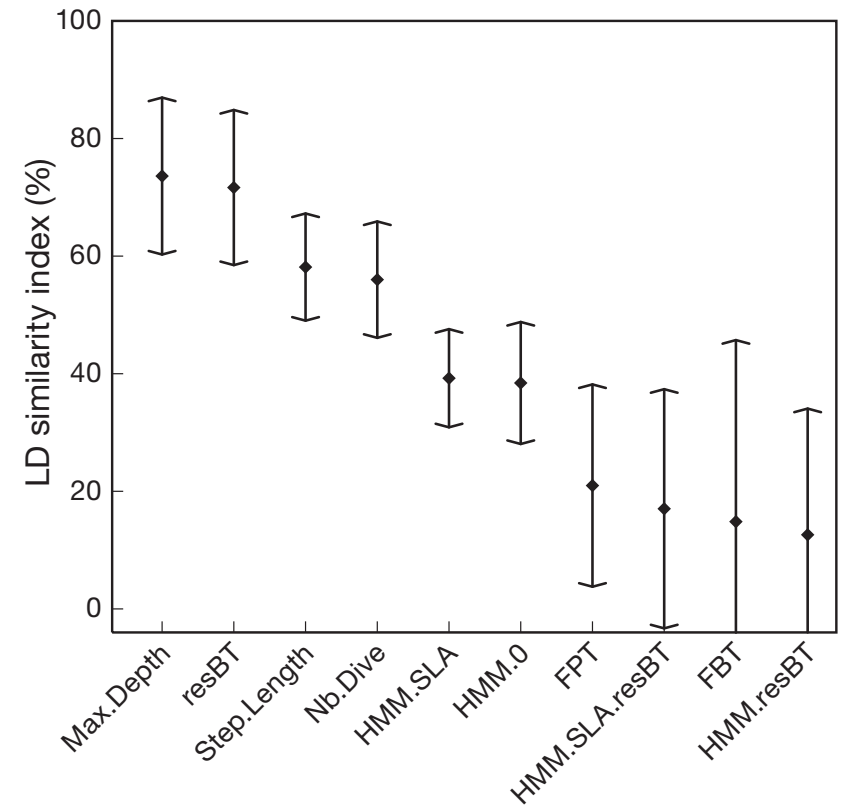

Fig. 3. Percentage of similarity, given by the Levenshtein distance (LD) similarity index (1 - LD), between track-based methods applied to Argos and GPS tracks of 6 southern elephant seals. Error bars are SD. Empirical descriptors presented the highest similarities (over 50\%) and 2 HMMs presented similarities close to $40 \%$. Max.Depth: maximum diving depth; resBT: bottom time residuals; Step.Length: step length; Nb.Dive: number of dives; FPT: first passage time; FBT: first bottom time

Table 2. Metabolisation time lags $( \pm \mathrm{SD})$ for southern elephant seals estimated for all track-based methods (descriptives and processbased ones) on Argos and GPS resolution datasets

\begin{tabular}{|lcc|}
\hline Methods & Argos & GPS \\
\hline Descriptive & & \\
Number of dives km ${ }^{-1}$ (Nb.Dive) & $3.33 \pm 1.15$ & $5.00 \pm 4.36$ \\
$\begin{array}{l}\text { Bottom time residuals (resBT) } \\
\text { Step length (Step.Length) }\end{array}$ & $3.67 \pm 3.79$ & $3.00 \pm 3.46$ \\
Maximum diving depth (Max.Depth) & $3.33 \pm 4.04$ & $6.33 \pm 1.15$ \\
First passage time (FPT) & $4.07 \pm 0.43$ & $9.67 \pm 1.53$ \\
First bottom time (FBT) & $5.00 \pm 1.05$ & $5.42 \pm 1.74$ \\
Mean & $4.12 \pm 0.86$ & $5.99 \pm 2.17$ \\
& & \\
Process-based (Hidden Markov Models) & \\
HMM.0 & $4.33 \pm 2.52$ & $4.67 \pm 5.51$ \\
$\quad$ (no covariate) & $4.33 \pm 4.04$ & $6.67 \pm 3.79$ \\
HMM.SLA & & \\
$\quad$ (environmental covariate) & $6.00 \pm 7.07$ & $7.00 \pm 5.29$ \\
HMM.resBT & & \\
$\quad$ (behavioural covariate) & $4.33 \pm 4.93$ & $6.33 \pm 4.62$ \\
HMM.SLA.resBT & & \\
$\quad$ (env. $\times$ behav. covariate) & $4.74 \pm 0.83$ & $6.37 \pm 0.63$ \\
Mean & & \\
Total mean & $\mathbf{4 . 2 7} \pm \mathbf{0 . 7 9}$ & $\mathbf{6 . 1 5} \pm \mathbf{1 . 6 7}$ \\
\hline
\end{tabular}

\section{Validity of ARS identification by the track-based methods compared to the drift rate index of foraging}

The LD similarity index and Jaccard index (results not shown) calculated with time lags from 0 to $12 \mathrm{~d}$ showed great variations between data resolutions. Table 2 presents time lags obtained for the various track-based methods. Argos data showed maximal similarity for an average lag of $4 \mathrm{~d}(4.27 \pm 0.79$, SD calculated across track-based methods) whereas similarity with GPS data was maximised for higher lags $(6.15 \pm 1.67)$. However, for the HMM with no covariate, the lag estimations with Argos and GPS tracks were very close: the time lag between the intensive foraging mode and the occurrence of positive change in drift rate was estimated at $4.33 \pm 2.52 \mathrm{~d}$ (SD across individuals) on Argos tracks and $4.67 \pm 5.51 \mathrm{~d}$ on GPS tracks. Estimations for FBT were also similar between Argos and GPS track analyses (Argos: $5.00 \pm$ 1.05 and GPS: $5.42 \pm 1.74$ ). Consequently, for the rest of the study, the minimal time lag of fat metabolisation was considered to be $4 \mathrm{~d}$ for post-breeding females. Fig. 4 illustrates the mean LD similarity index (and SD across individuals) between each trackbased method and the drift rate index with a $4 \mathrm{~d}$ time lag. Values for methods applied to Argos data (Fig. 4a) were of the same order as those for methods applied to GPS data (Fig. 4b, Argos: $33.29 \pm 18.13 \%$; GPS: $44.04 \pm 27.51 \%, t=-1.03$, $\mathrm{df}=15.5$, p-value $=0.31$ ). With Argos data, the highest similarities to the drift rate index of foraging were obtained between HMM.0 $(56.00 \%)$ and HMM.SLA (61.86\%). The 2 other HMMs presented high variations between individuals and lower values of similarity index than FBT and FPT (50.19 and $39.62 \%$, respectively). For GPS resolution, similarity to the drift rate index of foraging was maximised with HMM.O and HMM.SLA (71.23 and $70.45 \%$ ). Other track-based methods such as HMM with behavioural covariate (resBT), FPT and FBT presented high similarities with the drift rate index of foraging. Finally, the poorest similarities were obtained with methods based on empirical descriptors (Max.Depth: $15.42 \%$ with Argos and $11.04 \%$ with GPS tracking data). Fig. 5 illustrates various track-based methods including HMM.SLA, FPT, maximum diving depth and drift rate index of foraging (given a $4 \mathrm{~d}$ time lag) on the GPS track of 1 individual. The illustrated example depicts the range of similarities presented in Fig. 4: HMM with the 


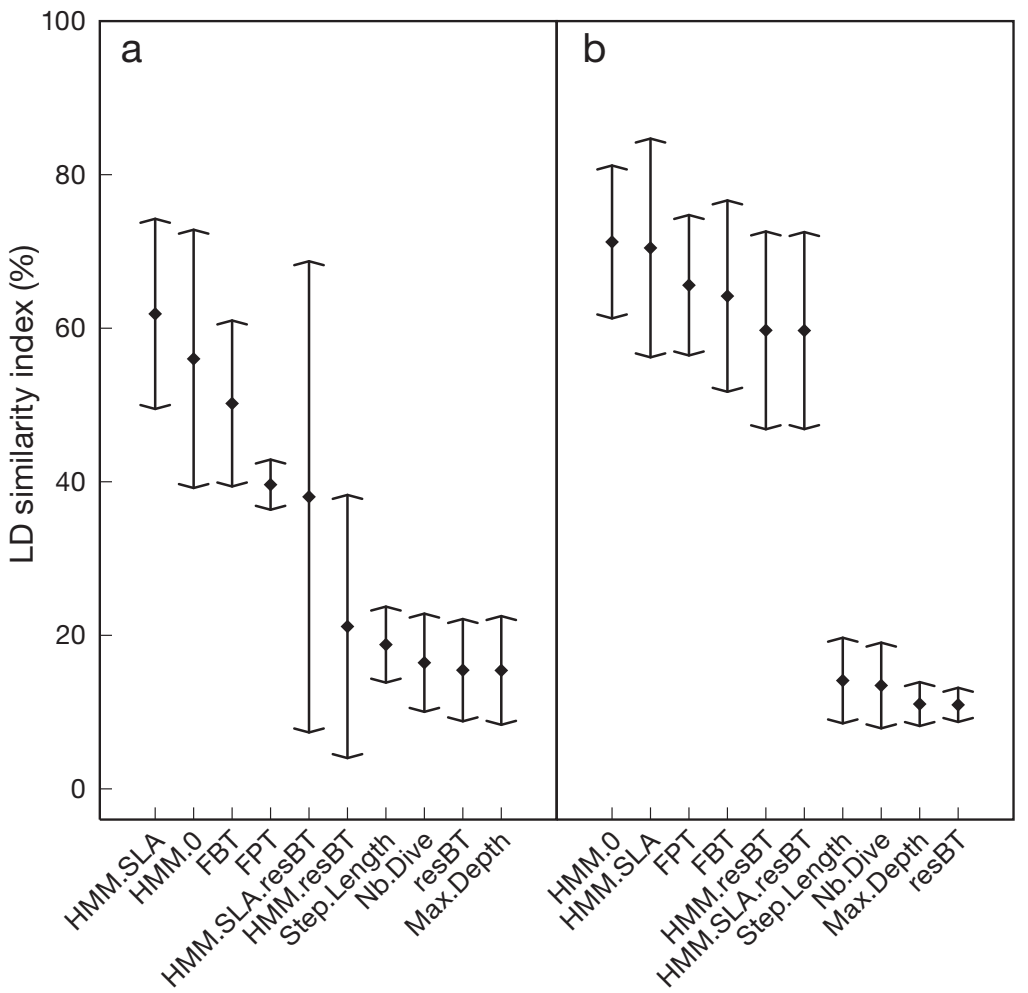

Fig. 4. Mean Levenshtein distance (LD) similarity index (1 - LD) calculated for all southern elephant seals (in \%) between the drift rate foraging index and the track-based methods on (a) Argos tracks and (b) GPS ones. A time lag of $4 \mathrm{~d}$ was included between the outputs of track-based methods and changes in drift rate foraging index. Error bars are SD. Abbreviations are as in Fig. 3

SLA covariate is the most similar, FPT and step length detect the main foraging areas, and the other empirical descriptors do not succeed in the detection of intensive areas compared to the ones highlighted by the drift rate foraging index.

\section{DISCUSSION AND PERSPECTIVES}

This study investigates the most common trackbased methods used to identify intensive foraging along predators' tracks. Six double-tagged seals were used for comparing the performances of methods on Argos versus GPS data. The methods' outputs were then related to an independent, and assumed true, index of foraging success determined from changes in the animal's buoyancy and hence body condition. Our results showed that the detection of ARS behaviour and intensive foraging effort can be equally assessed from low (Argos) and high (GPS) resolution tracking data. We also provided an estimation of the metabolisation time lag needed by the post-breeding seals to process their food and im- prove their body conditions. Given this time lag, intensive foraging effort has been directly associated with successful foraging behaviour leading to lipid anabolism and an increase in buoyancy. Finally, the HMM used here, with or without an environmental covariate, appeared as the best methodology among the tested methods to identify locations of actual successful foraging along the predators' tracks. These results have direct implications in the evaluation of predators' actual foraging and feeding ranges with respect to environmental conditions and resource availability.

An evaluation of similarity between track-based methods applied to Argos and GPS data resolutions indicated that the methods based on empirical descriptors are the most similar. Methods based on empirical descriptors are largely dependent on timestamps, not locations, whereas FPT/FBT methods are highly dependent on the spatial scale choice (Barraquand \& Benhamou 2008). Therefore, FPT/FBT methods might rank badly in this similarity evaluation due to the differences in spatial resolutions and scales between Argos and GPS track analyses. Unlike results of FPT and FBT, results of HMMs do not rely on spatial-scale choice. Intermediate similarities between Argos and GPS track analyses are therefore displayed by HMM methods, except for the HMM with resBT as covariate. However, caution must also be taken with process-based models since they are likely to be sensitive to spatial scale and data resolution (Breed et al. 2011). No change across subsampling frequency was observed in the proportion of time spent in intensive foraging along the tracks (see Fig. S2 in Supplement 1). GPS tracks include low error in the observations and so do the subsampled tracks from the GPS datasets. We can expect that similar subsamplings on Argos data, including high error observations, would lead to important changes between the proportions of intensive foraging observed along the subsampled tracks. However, the proportions of time spent in intensive foraging assigned by HMM.0 and HMM.SLA are globally consistent between low and high resolution tracking data. Consequently, both data resolutions can be considered adequate to identify intensive foraging effort from those process-based methods. However, 


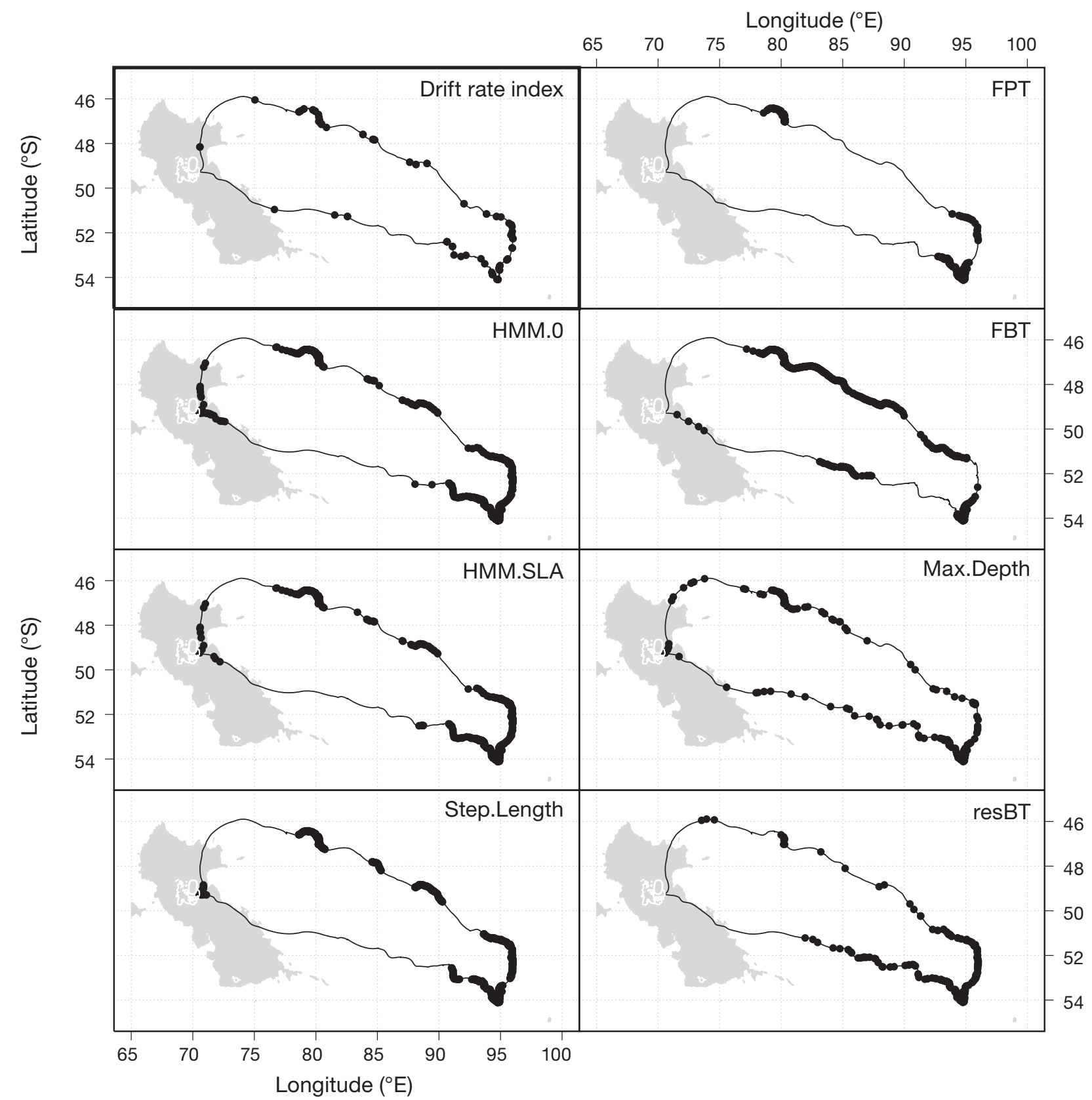

Fig. 5. Illustration of the outputs of several tested methods along a southern elephant seal track (same seal as in Fig. 1 and Fig. S1 in Supplement 1 at www.int-res.com/articles/suppl/m452p253_supp/). The top-left, black-framed, window is the drift rate foraging index: black dots correspond to the areas of extreme increases in drift rate. All the other windows are the outputs of track-based methods. A time lag of $4 \mathrm{~d}$ was included between the outputs of track-based methods and changes in the drift rate foraging index. Extensive foraging areas (thin black lines) correspond to straight fast-swimming areas, whereas intensive foraging areas (black dots) present area-restricted search characteristics (high sinuosity and slow displacement speed). The Kerguelen Plateau (bathymetry above $1000 \mathrm{~m}$ ) is shaded in grey while the Kerguelen Islands are depicted in white within the grey area. Abbreviations are as in Fig. 3

the species biology has to be taken into account, and there may be a difference between Argos and GPS data for predator species that make smaller moves or shorter trips (Breed et al. 2011).

The minimal metabolisation time lag was estimated to be $4 \mathrm{~d}$ based on a comparison between the output of track-based methods and the drift rate index of successful foraging. This minimal lag is in the range of values mentioned for post-breeding females in the literature and seems biologically coherent for mammals (Rosen et al. 2007, Thums et al. 2008). Although empirical descriptors presented high 
similarities in their outputs between Argos and GPS track analyses, very little similarity was obtained between their outputs and the drift rate index of successful foraging. In this case, consistency between data resolution was not a good indicator of the method's validity in ARS detection. Conversely, FPT and FBT presented high similarities between their outputs and the drift rate index of foraging but very low consistency between Argos and GPS analyses. In a spatially and temporally heterogeneous environment, predators can forage on patches of various sizes and densities. It is therefore expected that a foraging predator will display movement patterns at multiple spatial and temporal scales (Fauchald 1999). Firstly, the choice of one, and only one, spatial scale can be biased for FPT methods (Barraquand \& Benhamou 2008). Secondly, this choice potentially avoids the detection of ARS displayed at multiple scales (Orians \& Wittenberger 1991). In contrast, processbased models avoid this spatial scale choice and can thus detect multiple-scale intensively used areas along the tracks. Finally, HMMs do not require the rather strong assumption behind FPT methods of unbiased isotropic movements during intensive foraging (Fauchald \& Tveraa 2003).

In conclusion, some HMMs (HMM.0 and HMM. SLA) also combined a high level of consistency between Argos and GPS analyses with close similarities between their outputs and the drift rate index of foraging. They appeared to be the most optimal methods for the detection of successful foraging areas for both resolutions of tracking data. The models, both with and without an environmental covariate, insured an adequate segmentation with vague priors and a prior check of the underlying ARS hypothesis (that is, the bimodal distribution of movement parameters). Furthermore, given that the movement variables follow a Markov chain, the behavioural segments present a homogeneity that is consistent with the auto-correlated behaviour of a wild predator. In the present study, given the small number of iterations and computing time, convergence for the 2 models was quickly reached for all individuals. In regard to the 2 other HMMs, the bimodality of covariates (resBT and SLA) suggests that they would converge provided that there is a larger number of iterations in future analyses. DIC values were also the smallest for the 2 converging models compared to other HMMs, although the DIC statistics for HMM.SLA is almost double that of the simplest model. In the present study, the best methodological compromise to properly detect successful intensive foraging areas seemed to be the use of the least parametrised model: the HMM with no covariate. On the other hand, the use of an environmental covariate allowed a quantified estimation of the effect of SLA on the seals' foraging behaviour (through the coefficients $a 0$ and $a 1$ ): the probability of staying in intensive foraging behaviour was increased with negative SLA that characterise the structures of cyclonic eddies where the seals mainly forage (Bakun 2006, Bailleul et al. 2010, Dragon et al. 2010). Finally, HMM flexibility allows for the adaptation to biological characteristics of predator species: the model structure can be extended to 3 or more behavioural modes (Cappé et al. 2005), depending on the biology of the studied species, and relationships between predator's behaviour and their direct environment can be taken into account by the addition of covariates in the model (Eckert et al. 2008).

In the present study, the HMMs were applied to fixed time steps data due to a resampling that has potentially introduced a bias. However, the outputs evaluation by an independent methodology, the drift rate index, showed that this bias is negligible. Furthermore, we assumed that the filtered Argos and the GPS tracking data were low-error observations. Given such observations, HMMs displayed a high ability to identify foraging zones. It is likely though that filtered Argos data still contain observation errors. State-space models are characterised by an observation model dealing with the noisy raw data and a process model that can, as HMMs do, draw statistical inferences about foraging behaviours along the predators' tracks. Therefore, state-space modelling combines statistical robustness and predictive ability (Patterson et al. 2008). In comparison with the other tested methods, process-based models, applied to nil-error observations or raw data, appear to be the most optimal methodology in behavioural ecology. Although elephant seals have a large scale of movement, it can be assumed that this methodology can also be used on other predators' movements, with smaller spatial scales. It is our intention to examine this in future work. In the present study, differences in the temporal scales of detected events represent the main limitation: important changes in drift rate can only be detected over a few days, everything else being equal (Biuw et al. 2003). Therefore, drift rate data may not detect punctual successful foraging zones. However, by focusing on the most detectable changes in drift rates (90\% quantile), we adopted a conservative approach for the calculation of the metabolisation lag. Consequently, we assumed that the detection of drift rate changes provided valuable information on successful foraging zones at medium 
temporal and spatial scales. Such medium scales match the Argos resolution better than the fine GPS one, which may explain the differences observed between the 2 resolutions for the lag estimations. Furthermore, we focused the present study on the similarity between intensive foraging effort and changes in body condition. Further work could also take into account ARS that do not result in successive fat metabolisation and would therefore appear in trackbased methods' outputs but not in the drift rate successful foraging index considered here. In this case, accelerometry data would allow the quantification of foraging activity by, for instance, the study of flip stroke intensity (Wilson et al. 2007). The use of accelerometers would also enable the identification of seals' foraging success at finer temporal and spatial scales (Viviant et al. 2010).

\section{CONCLUSIONS}

In highly migratory marine species, which are impossible to directly observe across their range, existing information on the location of foraging and feeding behaviour is mainly inferred from surface movement analysis. The primary approach used to investigate movement has become telemetry-based methods that represent a rapidly growing field of research. Specific analysis methods have been developed for this purpose, taking into account spatial resolution of complex individual-based data (Jonsen et al. 2007, Bailleul et al. 2008 among others). This study showed that intensive foraging areas could be equally well detected from low and high resolution tracking datasets. HMM, a type of process-based models, were also shown to be the most efficient method to infer successful foraging behaviour from satellite tracking data of both resolutions. The favourable foraging zones identified by the models, both with and without an environmental covariate, were located where positive changes of the seals' body conditions occurred, given a 4 d metabolisation lag.

Tracking foraging activity with the GPS system produces new and valuable information about the fine-scale behaviour and the effort of predators in time and space, with more accurate and unbiased estimates than studies based on Argos telemetry. However, in the case of home-range studies, reserve design or large-scale environmental characterisation, Argos data resolution proved to be sufficient for the identification of the main successful foraging areas. Argos data resolution might therefore be pre- ferred by foraging ecologists for this range of questions. On the other hand, GPS resolution data, especially when combined with TDR data on diving behaviour, supports more precise investigations such as studies on fine temporal and spatial changes in the movement and diving patterns of predators in response to local oceanographic structures. Our approach was developed on southern elephant seal data, but the methods used and the results found in the present study have implications for foraging detection on telemetry data of other predator species fulfilling the ARS hypothesis.

Acknowledgements. The authors are grateful to Brigitte Planade, Matthieu Authier, Nory El Ksabi, Quentin Delorme and the field workers of Kerguelen 59th and 60th missions for their assistance in the field. This work was carried out in the framework of the IPSOS-SEAL (ANR VMC 07) program. We are indebted to IPEV (Institut Polaire Français), Total Foundation and TAAF (Terres Australes et Antarctiques Françaises) for financial support of Antarctic research program 109. We are also grateful to Pr Guihennec (MAP5, France) for her assistance in WinBUGS utilisation. Special thanks go to Tiphaine Jeanniard du Dot and 2 anonymous reviewers for very useful comments on a previous version of the manuscript.

\section{LITERATURE CITED}

Austin D, Bowen W, McMillan J, Boness D (2006) Stomach temperature telemetry reveals temporal patterns of foraging success in a free-ranging marine mammal. J Anim Ecol 75:408-420

Bailleul F, Charrassin J, Ezraty R, Girard-Ardhuin F, McMahon C, Field I, Guinet C (2007a) Southern elephant seals from Kerguelen Islands confronted by Antarctic Sea ice. Changes in movements and in diving behaviour. DeepSea Res II 54:343-355

Bailleul F, Charrassin J, Monestiez P, Roquet F, Biuw M, Guinet C (2007b) Successful foraging zones of southern elephant seals from the Kerguelen Islands in relation to oceanographic conditions. Philos Trans R Soc B 362: 2169-2181

> Bailleul F, Pinaud D, Hindell M, Charrassin J, Guinet C (2008) Assessment of scale-dependent foraging behaviour in southern elephant seals incorporating the vertical dimension: a development of the first passage time method. J Anim Ecol 77:948-957

> Bailleul F, Cotté C, Guinet C (2010) Mesoscale eddies as foraging area of a deep-diving predator, the southern elephant seal. Mar Ecol Prog Ser 408:251-264

Bakun A (2006) Fronts and eddies as key structures in the habitat of marine fish larvae: opportunity, adaptive response and competitive advantage. Sci Mar 70(S2): 105-122

Barraquand F, Benhamou S (2008) Animal movements in heterogeneous landscapes: identifying profitable places and homogeneous movement bouts. Ecology 89:3336-3348

> Bartumeus F, Catalan J, Viswanathan G, Raposo E, da Luz M (2008) The influence of turning angles on the success of non-orientated animal searches. J Theor Biol 252:43-55 
Benhamou S (2004) How to reliably estimate the tortuosity of an animal's path: straightness, sinuosity, or fractal dimension? J Theor Biol 229:209-220

Benhamou S, Bovet P (1989) How animals use their environment: a new look at kinesis. Anim Behav 38:375-383

Biuw M, McConnell B, Bradshaw C, Burton H, Fedak M (2003) Blubber and buoyancy: monitoring the body condition of free-ranging seals using simple dive characteristics. J Exp Biol 206:3405-3423

- Biuw M, Boehme L, Guinet C, Hindell M and others (2007) Variations in behaviour and condition of a southern ocean top predator in relation to in situ oceanographic conditions. Proc Natl Acad Sci USA 104:13705-13710

Block BA, Dewar H, Blackwell S, Williams T and others (2001) Migratory movements depth preferences and thermal biology of Atlantic bluefin tuna. Science 293: 1310-1314

> Bost C, Cotté C, Bailleul F, Cherel Y and others (2009) The importance of oceanographic fronts to marine birds and mammals of the southern oceans. J Mar Syst 78:363-376

Boyd I, Arnbom T (1991) Diving behaviour in relation to water temperature in the southern elephant seal: foraging implications. Polar Biol 2:259-266

Breed GA, Costa D, Goebel M, Robinson P (2011) Electronic tracking tag programming is critical to data collection for behavioral time-series analysis. Ecosphere 2:art10

> Brooks S, Gelman A (1998) General methods for monitoring convergence of iterative simulations. J Comput Graph Statist 7:434-455

Buckland S, Newman K, Thomas L, Koesters N (2004) Statespace models for the dynamics of wild animal populations. Ecol Model 171:157-175

> Buechner HK, Craighead FC Jr, Craighead JJ, Cote CE (1971) Satellites for research on free-roaming animals. BioScience 21:1201-1205

Cappé O, Moulines E, Ryden T (2005) Inference in Hidden Markov Models. Springer, New York, NY

> Charnov EL (1976) Optimal foraging, the marginal value theorem. Theor Popul Biol 9:129-136

> Crocker D, Le Boeuf B, Costa D (1997) Drift diving in female northern elephant seals: implications for food processing. Can J Zool 75:27-39

> Dale M (1989) Dissimilarity for partially ranked data and its application to overabundance data. Vegetatio 82:1-12

> Dennis B (1996) Discussion: Should ecologists become Bayesians? Ecol Appl 6:1095-1103

> Dicke M, Burrough P (1988) Using fractal dimensions for characterizing tortuosity of animal traits. Physiol Entomol 13:393-398

> Dragon AC, Monestiez P, Bar-Hen A, Guinet C (2010) Linking foraging behaviour to physical oceanographic structures: southern elephant seals and mesoscale eddies east of Kerguelen Islands. Prog Oceanogr 87:61-71

Eckert SA, Moore J, Dunn D, Sagarminaga van Buiten R, Eckert K, Halpin P (2008) Modelling loggerhead turtle movement in the Mediterranean: importance of body size and oceanography. Ecol Appl 18:290-308

Farina A, Lattanzi E, Malavasi R, Pieretti N, Piccioli L (2011) Avian soundscapes and cognitive landscapes: theory, application and ecological perspectives. Landsc Ecol 26:1257-1267

Fauchald P (1999) Foraging in a hierarchical patch system. Am Nat 153:603-613

Fauchald P, Tveraa T (2003) Using first-passage time in the analysis of area-restricted search and habitat selection.
Ecology 84:282-288

Fowler SL, Costa D, Arnould J, Gales N, Kuhn C (2006) Ontogeny of diving behaviour in the Australian sea lion: trials of adolescence in a late bloomer. J Anim Ecol 75: 358-367

Franke A, Caelli T, Hudson R (2004) Analysis of movements and behavior of caribou (Rangifer tarandus) using hidden Markov models. Ecol Model 173:259-270

- Gaspar P, Georges J, Fossette S, Lenoble A, Ferraroli S, Le Maho Y (2006) Marine animal behaviour: neglecting ocean currents can lead us up the wrong track. Proc Biol Sci 273:2697-2702

> Gelman A, Rubin D (1992) Inference from iterative simulation using multiple sequences. Stat Sci 7:457-511

- Girard C, Sudre J, Benhamou S, Roos D, Luschi P (2006) Homing in green turtles Chelonia mydas: oceanic currents act as a constraint rather than as an information source. Mar Ecol Prog Ser 322:281-289

Gurarie E, Andrews R, Laidre K (2009) A novel method for identifying behavioural changes in animal movement data. Ecol Lett 12:395-408

Jaccard P (1901) Étude comparative de la distribution florale dans une portion des Alpes et du Jura. Bull Soc Vaud Sci Nat 37:547-579 (in French)

> Jonsen I, Mills Flemming J, Myers R (2005) Robust statespace modeling of animal movement. Ecology 86: 2874-2880

> Jonsen I, Myers R, James M (2007) Identifying leatherback turtle foraging behaviour from satellite telemetry using a switching state-space model. Mar Ecol Prog Ser 337: 255-264

> Kareiva P, Odell G (1987) Swarms of predators exhibit 'preytaxis' if individual predators use area-restricted search. Am Nat 130:233-270

Le Boeuf B (1994) Variation in diving pattern of northern elephant seals with age, mass, sex, and reproductive condition. In: Le Boeuf BJ, Laws RM (eds) Elephant seals population ecology behaviour and physiology. University of California Press, Berkeley, CA, p 237-252

Levenshtein VI (1966) Binary codes capable of correcting deletions, insertions, and reversals. Sov Phys Dokl 10: $707-710$

McConnell B, Chambers C, Fedak M (1992) Foraging ecology of southern elephant seals in relation to the bathymetry and productivity of the southern ocean. Antarct Sci 4:393-398

McMahon CR, Burton H, McLean S, Slip D, Bester M (2000) Field immobilisation of southern elephant seals with intravenous tiletamine and zolazepam. Vet Rec 146: 251-254

Miller PJO, Johnson MP, Tyack PL (2004) Sperm whale behaviour indicates the use of echolocation click buzzes 'creaks' in prey capture. Proc Biol Sci 271:2239-2247

Morales J, Haydon D, Frair J, Holsinger K, Fryxell J (2004) Extracting more out of relocation data: building movement models as mixtures of random walks. Ecology 85: 2436-2445

Orians G, Wittenberger J (1991) Spatial and temporal scales in habitat selection. Am Nat 137:829-849

Orsi A, Whitworth T III, Nowlin J (1995) On the meridional extent and fronts of the Antarctic Circumpolar Current. Deep-Sea Res I 42:641-673

> Papadimitriou F (2009) Modelling spatial landscape complexity using the Levenshtein algorithm. Ecol Inform 4: $48-55$ 
Parker G, Stuart R (1976) Animal behaviour as a strategy optimizer: evolution of resource assessment strategies and optimal emigration thresholds. Am Nat 110:1055-1076

Patterson TA, Thomas L, Wilcox C, Ovaskainen O, Matthiopopoulos J (2008) State-space models of individual animal movement. Trends Ecol Evol 23:87-94

Patterson TA, McConnell B, Fedak M, Bravington M, Hindell M (2010) Using GPS data to evaluate the accuracy of state-space methods for correction of Argos satellite telemetry error. Ecology 91:273-285

R Development Core Team (2009) R: a language and environment for statistical computing. R Foundation for Statistical Computing, Vienna. www.R-project.org, ISBN3900051-07-0

Rosen DAS, Winship AJ, Hoopes LA (2007) Thermal and digestive constraints to foraging behaviour in marine mammals. Philos Trans R Soc B 362:2151-2168

Schick RS, Loarie S, Colchero F, Best B and others (2008) Understanding movement data and movement processes: current and emerging directions. Ecol Lett 11: $1338-1350$

Schmitt F, Seuront L (2001) Multifractal random walk in copepod behavior. Physica A 301:375-396

Spiegelhalter D, Thomas A, Best N (1999) WinBUGS Version 1.2 user manual. MRC Biostatistics Unit, Cambridge

Spiegelhalter D, Best N, Carlin B, Van der Linde A (2002) Bayesian measures of model complexity and fit. J R Stat Soc Series B Stat Methodol 64:583-616

Stevick P, McConnell B, Hammond P (2002) Patterns of movement. In: Hoelzel AR (ed) Marine mammal biology: an evolutionary approach. Blackwell Science, Oxford, p 185-216

Sturtz S, Ligges U, Gelman A (2005) R2winbugs: a package

Editorial responsibility: Rory Wilson,

Swansea, UK for running WinBUGS from R. J Stat Softw 12:1-16

Thums M, Bradshaw CJ, Hindell MA (2008) Tracking changes in relative body composition of southern elephant seals using swim speed data. Mar Ecol Prog Ser 370:249-261

Tremblay Y, Roberts AJ, Costa DP (2007) Fractal landscape method: an alternative approach to measuring arearestricted searching behaviour. J Exp Biol 210:935-945

Turchin P (1991) Translating foraging movements in heterogeneous environments into the spatial distribution of foragers. Ecology 72:1253-1266

> Turchin P (1996) Fractal analysis of animal movements: a critique. Ecology 77:2086-2090

- Vermard Y, Rivot E, Mahevas S, Marchal P, Gascuel D (2010) Identifying fishing trip behaviour and estimating effort from VMS data using Bayesian Hidden Markov Models. Ecol Model 221:1757-1769

> Viviant M, Trites A, Rosen D, Monestiez P, Guinet C (2010) Prey capture attempts can be detected in Steller sea lions and other marine predators using accelerometers. Polar Biol 33:713-719

> Walker E, Bez N (2010) A pioneer validation of a state-space model of vessel trajectories (VMS) with observers' data. Ecol Model 221:2008-2017

> Webb PM, Crocker DE, Blackwell SB, Costa DP, Le Boeuf BJ (1998) Effects of buoyancy on the diving behavior of northern elephant seals. J Exp Biol 201:2349-2358

White G, Garrott R (1990) Analysis of wildlife radio-tracking data. Academic Press, San Francisco, CA

> Wilson R, Liebsch N, Davies I, Quintana F and others (2007) All at sea with animal tracks; methodological and analytical solutions for the resolution of movement. Deep-Sea Res II 54:193-210

Submitted: July 11, 2011; Accepted: January 23, 2012

Proofs received from author(s): April 13, 2012 